\title{
Impact de programmes d'Activités de lecture interactives sur le développement cognitif et langagier d'enfants âgés de 0 à 5 ans
}

\author{
Les programmes ALI
}

The Impact of Interactive Reading Programs on Cognitive and

Language Development Among Children from 0 to 5 Years Old

The ALI Programs

\section{Impacto de programas de Actividades de Lectura Interactiva sobre el desarrollo cognitivo y lingüístico de los niños entre 0 y 5 años}

\section{Los programas ALI}

\section{Martine Verreault, Andrée Pomerleau et Gérard Malcuit}

Volume 33, numéro 2, automne 2005

Nouvelles tendances à l'égard de la petite enfance

URI : https://id.erudit.org/iderudit/1079107ar

DOI : https://doi.org/10.7202/1079107ar

Aller au sommaire du numéro

Éditeur(s)

Association canadienne d'éducation de langue française

ISSN

0849-1089 (imprimé)

1916-8659 (numérique)

Découvrir la revue

\section{Citer cet article}

Verreault, M., Pomerleau, A. \& Malcuit, G. (2005). Impact de programmes d'Activités de lecture interactives sur le développement cognitif et langagier d'enfants âgés de 0 à 5 ans : les programmes ALI. Éducation et francophonie, 33(2), 182-206. https://doi.org/10.7202/1079107ar

\section{Résumé de l'article}

La présente étude examine l'impact d'Activités de Lecture Interactive (ALI) sur le développement cognitif et langagier d'enfants d'âge préscolaire. Cent douze enfants bénéficient de l'intervention selon trois programmes (ALI-Bébé, ALI-Bambin et ALI-Explorateur). Les programmes sont implantés à domicile, en milieu de garde ou dans les deux contextes. Nous évaluons le développement cognitif et langagier des enfants avant le début des programmes, puis après 6 mois d'application. Dans l'ensemble, les enfants obtiennent des résultats normalisés significativement plus élevés à la deuxième mesure. Nous observons une différence entre les programmes : les résultats de développement augmentent de façon significative pour les enfants des programmes ALI-Bébé et ALI-Bambin, mais non pour ceux du programme ALI-Explorateur. La grandeur des gains ne diffère pas d'un contexte d'implantation à l'autre. De plus, nos résultats révèlent que l'intervention entraîne des gains significatifs aux mesures de langage expressif et d'habiletés non-verbales. La discussion s'organise autour de ces constatations. Notre étude souligne l'efficacité de programmes axés sur des Activités de Lecture Interactive pour hausser le développement cognitif et langagier des enfants durant les années préscolaires.
Tous droits réservés (C) Association canadienne d'éducation de langue française, 2005
Cecument est protégé par la loi sur le droit d'auteur. L'utilisation des services d'Érudit (y compris la reproduction) est assujettie à sa politique d'utilisation que vous pouvez consulter en ligne. 


\section{Impact de programmes d'Activités de lecture interactives sur le développement cognitif et langagier d'enfants âgés de 0 à 5 ans : les programmes ALI}

\section{Martine VERREAULT}

Université du Québec à Montréal, Québec, Canada

Andrée POMERLEAU

Université du Québec à Montréal, Québec, Canada

\section{Gérard MALCUIT}

Université du Québec à Montréal, Québec, Canada

\section{RÉSUMÉ}

La présente étude examine l'impact d'Activités de Lecture Interactive (ALI) sur le développement cognitif et langagier d'enfants d'âge préscolaire. Cent douze enfants bénéficient de l'intervention selon trois programmes (ALI-Bébé, ALI-Bambin et ALI-Explorateur). Les programmes sont implantés à domicile, en milieu de garde ou dans les deux contextes. Nous évaluons le développement cognitif et langagier des enfants avant le début des programmes, puis après 6 mois d'application. Dans 
l'ensemble, les enfants obtiennent des résultats normalisés significativement plus élevés à la deuxième mesure. Nous observons une différence entre les programmes : les résultats de développement augmentent de façon significative pour les enfants des programmes ALI-Bébé et ALI-Bambin, mais non pour ceux du programme ALIExplorateur. La grandeur des gains ne diffère pas d'un contexte d'implantation à l'autre. De plus, nos résultats révèlent que l'intervention entraîne des gains significatifs aux mesures de langage expressif et d'habiletés non-verbales. La discussion s'organise autour de ces constatations. Notre étude souligne l'efficacité de programmes axés sur des Activités de Lecture Interactive pour hausser le développement cognitif et langagier des enfants durant les années préscolaires.

\section{ABSTRACT}

\section{The Impact of Interactive Reading Programs on Cognitive and Language Development Among Children from 0 to 5 Years Old: The ALI Programs}

Martine VERREAULT, Andrée POMERLEAU and Gérard MALCUIT

Infant Study Lab, Department of Psychology

Université du Québec à Montréal, Québec, Canada

This study examines the impact of interactive reading activities (ALI) on the cognitive and language development of pre-school-aged children. One hundred twelve children benefit from the interventions of three programs (ALI-Bébé, ALI-Bambin et ALI-Explorateur). These programs are established in the home, in day-care centres or in both contexts. We evaluate the cognitive and language development of children before they begin the programs, and again after 6 months of application. Overall, the children's standardized scores were significantly higher after the programs. We observed a difference between the programs - the development scores increase significantly for children in the ALI-Bébé and ALI-Bambin programs, but not for those in the ALI-Explorateur program. The size of gains does not differ from one settingcontext to another. Our results also reveal that the intervention leads to significant gains in expressive language and non-verbal abilities scores. The discussion revolves around these findings. Our study highlights the effectiveness of programs based on interactive reading activities as a means of raising the cognitive and language development of children during the pre-school years. 


\section{RESUMEN}

\section{Impacto de programas de Actividades de Lectura Interactiva sobre el desarrollo cognitivo y lingüístico de los niños entre 0 y 5 años : los programas ALI}

Martine Verreault, Andrée Pomerleau y Gérard Malcuit

Laboratorio de estudios sobre los lactantes, Departamento de sicología

Universidad de Quebec en Montreal, Quebec, Canadá

El presente estudio examina el impacto de las actividades de lectura interactiva (ALI) sobre el desarrollo cognitivo y lingüístico de los niños en edad preescolar. Ciento doce niños beneficiarios de dicha intervención según tres programas (ALIBebé, ALI-Chiquillo, y ALI-Explorador). Los programas se llevan a cabo en los domicilios, en las guarderías o en los dos contextos. Evaluamos el desarrollo cognitivo y lingüístico de los niños antes del inicio del programa y 6 meses después de su implantación. En total, los niños obtienen resultados normalizados significativamente más elevados durante el segundo programa. Observamos una diferencia entre los programas : los resultados del desarrollo aumentan de manera significativa para los niños de los programas ALI-Bebé y ALI-Chiquillo, pero no para los del programa ALI-Explorador. La proporción de lo adquirido no difiere de un contexto de implantación al otro. Además, nuestros resultados revelan que la intervención provoca ganancias significativas cuando se mide el lenguaje expresivo y las habilidades no-verbales. La discusión se organiza alrededor de estas constataciones. Nuestro estudio subraya la eficacia de los programas centrados en actividades de lectura interactiva para aumentar el desarrollo cognitivo y lingüístico de los niños durante los años preescolares.

De tels constats soulignent l'importance pour l'enfant d'acquérir tôt un bon niveau d'habiletés cognitives et langagières.

\section{Contexte théorique}

Les cinq premières années de vie de l'enfant semblent cruciales pour l'acquisition des habiletés nécessaires pour rencontrer les exigences des apprentissages scolaires. Certaines des compétences cognitives acquises avant la maternelle prédisent la performance scolaire (Ramey \& Ramey, 1998; Stevenson \& Newman, 1986). En effet, dans l'ensemble de ses compétences cognitives, le niveau des habiletés langagières de l'enfant d'âge préscolaire explique en bonne partie la qualité de son fonctionnement ultérieur à l'école (Scarborough \& Dobrich, 1994; Whitehurst, Epstein et al., 1994). Par exemple, l'étendue de son vocabulaire avant l'entrée à l'école est un bon prédicateur de son succès en lecture un peu plus tard (Wells, 1987). De tels constats soulignent l'importance pour l'enfant d'acquérir tôt un bon niveau d'habiletés cognitives et langagières. 
Un faible niveau cognitif et langagier à l'entrée à l'école handicape grandement le rendement scolaire ultérieur des enfants.
Dès leurs premières années de vie, les enfants présentent des différences dans la qualité de leur développement cognitif et langagier (Alexander \& Entwisle, 1988; Edwards, 1995). Selon un rapport de la Carnegie Foundation for the Advancement of Teaching (1991), 35 \% des jeunes américains entrent à la maternelle avec un niveau langagier insuffisant pour aborder avec succès les tâches d'apprentissage. Ils présentent des lacunes sur le plan du vocabulaire et de l'organisation grammaticale des phrases. Une enquête plus récente auprès d'enseignantes de la maternelle confirme cette réalité. Elle révèle que près de la moitié (48\%) des enfants américains réussissent mal la transition de la maison à l'école (National Center for Early Development and Learning, 1998). Le personnel rapporte que les enfants ont de la difficulté à comprendre des consignes, à travailler de façon autonome, à communiquer et à maîtriser des notions générales. Ces problèmes posent un défi, car il semble que ce que l'on observe à la maternelle se maintient jusqu'au secondaire (Stevenson \& Newman, 1986; Wells, 1987). Ainsi, un faible niveau cognitif et langagier à l'entrée à l'école handicape grandement le rendement scolaire ultérieur des enfants. L'enrichissement de leurs habiletés préscolaires faciliterait la transition à l'école et un meilleur succès par la suite (Alexander \& Entwisle, 1988).

Les expériences vécues pendant l'enfance, à la maison ou en milieu de garde, sont d'importantes sources d'influence pour le développement des habiletés langagières nécessaires aux apprentissages ultérieurs (Hart \& Risley, 1995). Selon l'approche interactionniste, le langage expressif et réceptif se développe, dès le début de la vie, à travers des expériences d'interactions offertes par les environnements familial et social de l'enfant (Ninio, 1992; Perna, 2003; Ramey \& Ramey, 1998; Snow, 1984). Les personnes présentes dans les milieux que fréquente l'enfant (parents, grandsparents, éducatrices, etc.) peuvent faciliter ou non son développement langagier.

Les échanges verbaux entre l'adulte et l'enfant contribuent également au développement du langage expressif et réceptif. L'enfant apprend le langage et les règles de la communication à partir de ce qu'il entend (Ninio, 1992). La recherche montre que la fréquence des interactions verbales entre les parents et leur bambin est en relation positive avec l'acquisition du vocabulaire de l'enfant à 2 ans (Huttenlocher, Haight, Bryk, Seltzer, \& Lyons, 1991) et avec son développement cognitif à 9 ans (Hart \& Risley, 1995). Cependant, la fréquence ne peut expliquer, à elle seule, le niveau de développement de l'enfant (Hart, 1991). Le style et le contenu langagier de l'adulte qui interagit avec lui doivent aussi être pris en considération. Lorsque le parent parle à son enfant, il adopte un style différent de celui qu'il utilise pour parler à un adulte. Il recourt à un langage simple et redondant, habituellement ajusté au niveau de compréhension du petit. L'étude de Hoff-Ginsberg (1985) confirme la relation positive entre le développement langagier de l'enfant et l'utilisation par le parent de phrases courtes, énoncées clairement avec une intonation expressive. De plus, des catégories d'énoncés présentes dans le langage de l'adulte sont particulièrement importantes pour expliquer le niveau langagier de l'enfant, notamment les énoncés interrogatifs et ceux qui renforcent les expressions (Lacroix, Pomerleau, \& Malcuit, 2002). En effet, les questions suscitent des réponses verbales de l'enfant. Qui plus est, le renforcement des sons qu'il produit, par des encouragements et des 
rétroactions, incite le bébé à continuer à faire ces sons et dirige ses productions vers des formes de plus en plus langagières (Malcuit, Pomerleau, \& Séguin, 2003). En somme, l'acquisition du langage par le jeune enfant dépend, en partie, de la quantité de ses échanges verbaux avec un adulte familier, du style langagier adopté par ce dernier et du type d'énoncés qu'il émet pendant l'interaction verbale (Ninio, 1992; Snow, 1984).

Des contextes d'interactions entre l'adulte et l'enfant sont plus propices que d'autres à de bons échanges. En particulier, les contextes de lecture permettent d'intégrer, de façon optimale, un style et un contenu associés à la qualité du développement langagier des enfants. En situation de lecture, l'adulte utilise une plus grande variété de mots et questionne plus souvent l'enfant que lors du jeu libre (Lewis \& Gregory, 1987). Il donne davantage de rétroactions et d'encouragements aux verbalisations de l'enfant que dans les autres contextes (McDonnell, Rollins, \& Friel-Patti, 2001). Les conduites verbales de l'adulte sont plus contingentes à celles de l'enfant et davantage associées à son développement langagier que celles présentes lors des soins, des repas, de l'habillement ou des jeux (Hoff-Ginsberg, 1991). L'adulte offre, en situation de lecture, un modèle langagier plus riche et plus varié que lors des autres activités.

De nombreuses études montrent l'importance de la lecture adulte-enfant et des conduites verbales qu'elle favorise. Le fait de regarder des livres avec un parent ou un adulte familier est positivement corrélé aux habiletés langagières de l'enfant (Bus, van IJzendoorn, \& Pellegrini, 1995; Scarborough \& Dobrich, 1994). Le lien entre les activités de lecture au cours des années préscolaires et le développement cognitif et langagier de l'enfant perdure. Crain-Thoreson et Dale (1992) rapportent une corrélation positive entre des sessions de lecture parent-enfant à 2 ans et des mesures cognitives et langagières prises à 2 et 4 ans. Aussi, des enfants ayant bénéficié d'une lecture régulière à l'âge préscolaire obtiennent à 13 ans des résultats plus élevés en lecture et aux tests de QI que ceux d'enfants ayant eu peu d'expériences de lecture (Stevenson \& Fredman, 1990).

La relation entre les activités de lecture et la qualité du langage de l'enfant est encore plus importante lorsque ces activités débutent dès le plus jeune âge (Scarborough \& Dobrich, 1994). L'âge de l'enfant au moment où les parents commencent les activités de lecture avec lui est un meilleur prédicateur de son niveau de langage à 2 ans que leur fréquence ou le nombre d'histoires racontées (DeBaryshe, 1993). Également, les enfants qui ont bénéficié davantage de lecture depuis l'âge de 1 an manifestent plus d'intérêt pour les livres et présentent de meilleures habiletés langagières à 2 ans (Lyytinen, Laakso, \& Poikkeus, 1998).

En résumé, la recherche indique que faire la lecture avec les enfants favorise leur développement cognitif et langagier, les prépare à devenir eux-mêmes des lecteurs (Wells, 1987) et facilite leur entrée dans le monde scolaire (Bus et al., 1995). Par ailleurs, la façon de faire la lecture avec le jeune enfant importe elle aussi. Un mode spécifique d'interactions durant la lecture se révèle particulièrement efficace. Whitehurst et ses collègues (1988) ont conçu un programme dit de lecture dialogique qui enseigne aux adultes (parents ou éducatrices) des techniques à utiliser lorsqu'ils 
Avec la lecture dialogique, l'adulte délaisse son rôle de locuteur pour devenir un auditeur actif. L'enfant apprend à raconter l'histoire. regardent des livres d'images avec un jeune enfant. Les techniques consistent à l'aider à préciser ses descriptions d'images, à corriger ses essais et à lui poser des questions afin de maintenir le débit de sa conversation et son intérêt. L'adulte renforce les verbalisations de l'enfant en réagissant de façon positive et en ajoutant de l'information appropriée. Ce mode d'interaction diffère de la lecture traditionnelle où l'enfant demeure silencieux pendant qu'on lui raconte une histoire. Avec la lecture dialogique, l'adulte délaisse son rôle de locuteur pour devenir un auditeur actif. L'enfant apprend à raconter l'histoire.

Les études de Whitehurst et de ses collègues soulignent l'efficacité de leur intervention auprès d'enfants âgés de 2 à 4 ans, de milieux socio-économiques et d'origines culturelles variés, tant à la maison qu'en milieu de garde (Lonigan \& Whitehurst, 1998; Valdez-Menchaca \& Whitehurst, 1992; Whitehurst et al., 1988; Whitehurst, Arnold et al., 1994; Whitehurst, Epstein et al., 1994). Les enfants qui participent à au moins 3 périodes hebdomadaires de lecture dialogique de 10 minutes chacune pendant 4 à 7 semaines ont des performances langagières supérieures à celles d'enfants d'un groupe contrôle. Les résultats indiquent des gains significatifs aux mesures de langage expressif. Aux mesures de langage réceptif, les résultats vont généralement dans le même sens, sans toutefois atteindre le seuil usuel de signification statistique.

Au Québec, des programmes d'Activités de Lecture Interactive (ALI) ont été mis en place dans une municipalité de la Montérégie. Il s'agit de trois programmes distincts, inspirés de la lecture dialogique, s'adressant aux enfants âgés de 0 à 5 ans. Ils ont pour objectif général de favoriser leur développement cognitif et langagier, et de les préparer aux apprentissages scolaires. Les activités des programmes ALI peuvent être réalisées par les parents ou les éducatrices en milieu de garde et s'adaptent aux habiletés croissantes des enfants. Pour le nourrisson, les activités ALI-Bébé stimulent le développement d'habiletés cognitives générales, comme l'attention et le début de la communication. Celles-ci l'amèneront, vers 15 mois, aux activités de lecture interactive du programme ALI-Bambin. Les techniques pour réaliser les activités de lecture interactive consistent, entre autres, à capter l'attention de l'enfant, à identifier les éléments d'une image, à formuler des questions ouvertes, à évaluer et à renforcer ses verbalisations. ALI-Bambin vise le développement des habiletés langagières expressives et réceptives. Enfin, vers 3 ans, ALI-Explorateur met l'accent sur les concepts à intégrer aux activités de lecture interactive pour favoriser l'acquisition des habiletés cognitives nécessaires aux apprentissages scolaires.

\section{Objectifs et hypothèses de recherche}

L'objectif général de l'étude est d'évaluer l'impact des programmes ALI sur le développement cognitif d'enfants d'âge préscolaire selon deux moments de mesure. L'évaluation se réalise à partir d'instruments de mesure standardisés donnant des résultats normalisés. Nous évaluons l'impact des trois programmes ALI après six mois d'application. L'impact devrait se traduire par des résultats normalisés plus élevés au deuxième moment de mesure qu'au premier. Nous examinons aussi l'im- 
pact selon le contexte d'implantation des programmes: à domicile, en milieu de garde ou dans les deux contextes. Nous attendons des gains plus importants chez les enfants qui reçoivent les programmes à la fois à domicile et en milieu de garde, puisqu'ils sont plus fréquemment exposés aux activités. Comme ALI vise de façon particulière à favoriser le développement langagier, nous distinguons dans la mesure du développement cognitif les habiletés langagières et non-langagières des enfants. Nous prévoyons une hausse plus importante des résultats aux mesures langagières.

\section{Méthode}

\section{Participants et participantes}

Les programmes ALI sont implantés dans une municipalité rurale de la Montérégie. La population de cette municipalité doit composer avec un facteur d'éloignement des services. Les problématiques présentes dans la région (sous-scolarisation, problèmes de langage chez les enfants) et le nombre élevé d'enfants âgés de 0 à 5 ans justifient une intervention préventive favorisant le développement cognitif et langagier de tous les enfants (Pomerleau, Malcuit, Laberge, \& Flynn, 1997). Divers moyens (annonces dans les journaux locaux et les milieux de garde, bouche à oreille, activités de promotion au CLSC et à la bibliothèque, etc.) permettent de rejoindre un grand nombre de familles, de présenter les programmes et de donner des informations sur l'étude. Les parents signent un formulaire de consentement pour la participation de l'enfant aux programmes et pour son évaluation. Ce formulaire décrit l'étude, assure la confidentialité des données recueillies et le droit de se retirer en tout temps sans avoir à le justifier.

Nous recrutons 335 enfants auprès desquels nous réalisons une première évaluation. De ceux-ci, nous retenons les 189 enfants qui participent à une deuxième évaluation 6 mois plus tard. Cette dernière permet de comparer les résultats des enfants avant le début des programmes et après 6 mois d'application. Nous ne retenons pas les données de 39 de ces enfants : 19 reçoivent un programme ALI adapté aux difficultés cognitives ou orthophoniques que la première évaluation a permis de détecter, 5 ne reçoivent aucune forme de programme entre les deux évaluations, 8 n'ont pu être évalués au tout début de leur participation, 1 est évalué la seconde fois dans un délai de 18 mois, 6 n'ont pas le même outil d'évaluation aux deux mesures. De plus, nous ne considérons pas, dans les analyses, les données de 38 autres enfants par manque d'assiduité d'application. Les enfants doivent participer aux programmes un minimum de trois mois pour être inclus dans l'étude d'évaluation.

Le groupe final de 112 enfants se compose de 59 filles et 53 garçons. Leur âge varie de 2,3 à 65,6 mois. Cinquante-cinq d'entre eux (49,1\%) fréquentent un centre de la petite enfance (CPE). Comme 18 des enfants ont une sœur ou un frère qui participe à l'étude, nous avons un échantillon de 94 familles. La scolarité moyenne des mères correspond à une année post-secondaire et celle des conjoints à un secondaire 5 . Cinquante-trois pour cent des familles ont un revenu annuel entre 30001 et $60000 \$$, $26,5 \%$ ont un revenu inférieur à $30001 \$$ et $20,5 \%$ un revenu supérieur à $60000 \$$. 
L'échantillon comprend trois groupes d'enfants répartis selon l'âge dans un programme particulier (ALI-Bébé, ALI-Bambin et ALI-Explorateur). Le premier groupe est formé de 37 bébés âgés de moins de 15 mois ( $M=5,5$ mois, étendue : 2,3 - 12,2 mois). Le deuxième groupe est constitué de 31 bambins âgés en moyenne de 23,9 mois (étendue : 12,4 - 53,2 mois). Le dernier groupe comprend 44 enfants âgés en moyenne de 51,1 mois (étendue : 31,7 - 65,6 mois). Des tests Khi-carré (_2) sur les caractéristiques des participants selon les trois programmes révèlent que la répartition des sexes, le revenu familial annuel et le niveau de scolarité de la mère et du conjoint ne se distinguent pas entre les groupes. Cependant, l'âge des mères et de leurs conjoints diffère, $F(2,99)=5,62, p<0,01$ et $F(2,93)=4,65, p<0,05$, respectivement. Le test a posteriori Student-Newman-Keuls précise que les mères et les conjoints du groupe ALI-Bébé sont plus jeunes que ceux du groupe ALI-Explorateur. Également, les enfants ALI-Bébé sont moins nombreux à fréquenter un CPE que les autres enfants ${ }_{-}^{2}(2, N=85)=13,83, p<0,01$. Les principales caractéristiques sociodémographiques des participants apparaissent au Tableau 1.

Tableau 1 : Caractéristiques des enfants et de leurs parents par programme

\begin{tabular}{|c|c|c|c|c|c|c|c|c|c|}
\hline & \multicolumn{3}{|c|}{$\begin{array}{l}\text { ALI - Bébé } \\
(\mathrm{n}=37)\end{array}$} & \multicolumn{3}{|c|}{$\begin{array}{c}\text { ALI-Bambin } \\
(n=31)\end{array}$} & \multicolumn{3}{|c|}{$\begin{array}{l}\text { ALI- Explorateur } \\
(n=44)\end{array}$} \\
\hline & M & É & $\%$ & M & É & $\%$ & M & ÉT & $\%$ \\
\hline Sexe de l'enfant (filles) & \multicolumn{3}{|r|}{54,1} & \multicolumn{3}{|r|}{48,4} & \multicolumn{3}{|r|}{54,5} \\
\hline Âge de l'enfant (en mois) & 5,5 & 2, & & 23,9 & 9, & & 51,1 & 9,4 & \\
\hline Enfants fréquentant un CPE & \multicolumn{3}{|r|}{13,5} & \multicolumn{3}{|r|}{67,7} & \multicolumn{3}{|r|}{65,9} \\
\hline Âge de la mère (en année) & 28,6 & 5 , & & 30,8 & 6 , & & 33,1 & 5,2 & \\
\hline Âge du conjoint & 31,7 & 7, & & 34,3 & 6 , & & 36,5 & 5,6 & \\
\hline Scolarité de la mère (en année) & 12,6 & 2, & & 13,0 & 2, & & 11,9 & 2,3 & \\
\hline Scolarité du conjoint & 11,1 & 2, & & 12,1 & 2 & & 11,5 & 2,5 & \\
\hline \multicolumn{10}{|l|}{ Revenu familial } \\
\hline$\leq 30000 \$$ & & & 21,9 & & & 16,0 & & & 34,3 \\
\hline $30001 \$-40000 \$$ & & & 21,9 & & & 20,0 & & & 25,7 \\
\hline $40001-60000 \$$ & & & 37,5 & & & 32,0 & & & 25,7 \\
\hline$\geq 60001 \$$ & & & 18,8 & & & 32,0 & & & 14,3 \\
\hline
\end{tabular}

\section{Programmes ALI}

\section{Programme ALI-Bébé : 0-15 mois}

Le programme ALI-Bébé propose des activités de stimulation susceptibles de favoriser le développement cognitif du tout-petit, en particulier l'attention, la communication et le langage. Les activités sont réparties en cinq volets (A, B, C, D, E) selon l'âge. Les volets présentent des activités telles parler à l'enfant lors des routines quotidiennes, lui faire suivre des yeux un objet, lui décrire les parties du corps, favoriser l'imitation d'une comptine, d'une séquence de gestes ou le cri des animaux, 
encourager les sons qu'il produit, explorer et manipuler un livre avec lui, etc. Avec le programme ALI-Bébé, l'enfant apprend à devenir attentif aux personnes et aux objets, il entend un adulte lui parler, il apprend à communiquer et à vocaliser, et il associe les images et les mots. ALI-Bébé favorise aussi la mise en place d'une routine incluant beaucoup de stimulations vocales et de contacts physiques chaleureux entre l'adulte et l'enfant. Ces moments d'activités le préparent à la lecture interactive proprement dite avec le parent ou l'éducatrice en milieu de garde.

\section{Programme ALI-Bambin : 15-36 mois}

Le programme ALI-Bambin montre aux adultes des techniques spécifiques à utiliser lorsqu'ils font les activités de lecture interactive avec l'enfant, telles obtenir son attention et utiliser son intérêt, nommer les éléments d'une image, répéter et encourager, poser des questions ouvertes et générales, ajouter de l'information, faire des liens avec la vie courante et établir le tour de parole. Ces techniques incitent l'enfant à verbaliser et à élaborer à partir de ce qu'il voit et de ce qu'il connaît. Elles sont réparties en quatre volets $(\mathrm{A}, \mathrm{B}, \mathrm{C}, \mathrm{D})$ de complexité croissante qui s'adaptent à la progression de l'enfant. Avec ALI-Bambin, l'adulte amène l'enfant à échanger avec lui. Il l'aide à préciser ses descriptions d'images et il lui pose des questions afin de maintenir le débit de sa conversation et son intérêt. L'adulte corrige et renforce les réponses de l'enfant par des encouragements, des répétitions et des expansions de ses énoncés. L'enfant élargit son vocabulaire et améliore sa prononciation en associant les images et les mots, et en nommant des objets, des actions ou des personnages.

\section{Programme ALI-Explorateur : 3-5 ans}

Le programme ALI-Explorateur met l'accent sur les connaissances et concepts à intégrer aux activités de lecture interactive pour favoriser l'acquisition des habiletés cognitives nécessaires aux apprentissages ultérieurs. Un volet préparatoire (volet Début) initie les adultes aux techniques de lecture interactive proprement dites. $\mathrm{Ce}$ volet correspond au dernier d'ALI-Bambin (volet D). ALI-Explorateur comprend quatre volets (A1, A2, B1, B2) présentant un ensemble de connaissances et de concepts qui peuvent être abordés lors des activités de lecture interactive. Le volet Al touche des catégories conceptuelles concrètes faisant référence au nom des animaux domestiques, au nom de leurs petits, aux fruits et aux légumes, au nom des principales parties du corps et à leurs fonctions. A2 porte sur des concepts plus abstraits, tels les mots utilisés dans les interrogations, les formes, les couleurs simples, les notions de temps, de quantité ou de grandeur, etc. Les volets B1 et B2 présentent des concepts similaires à ceux des volets $\mathrm{A}$, mais à un degré de complexité plus avancé. Par exemple, les adultes font référence aux animaux sauvages, de la jungle ou de la savane, aux fruits et légumes exotiques et aux nuances de couleur. 


\section{Déroulement}

Nous planifions, au domicile ou au CPE, un premier rendez-vous d'évaluation avec l'enfant avant la formation aux programmes ALI. Par la suite, des agentes de recherche réalisent les visites de formation, auprès des parents et des personnes présentes à la maison (grands-parents, fratrie) ou auprès des éducatrices en milieu de garde. Lors de chaque séance de formation, d'une durée d'environ une heure, l'agente explique les activités du volet en question et en fait la démonstration. Elle demande ensuite aux adultes de les mettre en pratique. Au besoin, elle donne des explications supplémentaires. Pour chaque volet d'ALI-Bambin, une vidéo d'environ 30 minutes illustre les techniques à adopter lors des activités de lecture interactive. Pour le volet Début d'ALI-Explorateur, une vidéo correspondant au dernier volet d'ALI-Bambin montre l'ensemble des techniques de lecture interactive. Par contre, les volets A1, A2, B1 et B2 d'ALI-Explorateur et les cinq volets d'ALI-Bébé n'ont pas de vidéo de formation. Trois semaines après la séance de formation, une visite de consolidation (à domicile ou en milieu de garde) permet de vérifier la compréhension des techniques, d'apporter des corrections au besoin et de renforcer les apprentissages des adultes. Les agentes assurent un suivi téléphonique mensuel pour examiner la réalisation des activités ou l'application des techniques de lecture et pour colliger l'information sur la fréquence et la durée des sessions ALI. On demande aux adultes de réaliser les activités de lecture ALI-Bambin ou ALI-Explorateur au moins 3 fois par semaine, pendant des sessions de 10 à 15 minutes chacune. Les activités ALI-Bébé sont plus difficilement quantifiables, car elles s'intègrent à la routine quotidienne de l'enfant.

\section{Matériel}

Les parents et les éducatrices reçoivent un manuel explicatif résumant et illustrant les activités du programme ALI-Bébé, les techniques de lecture interactive du programme ALI-Bambin ou les concepts et connaissances du programme ALIExplorateur.

Lors des séances de formation aux volets d'ALI-Bébé, nous donnons divers objets favorisant la stimulation, tels des marionnettes, des livres plastifiés, un hochet, etc. Pour ALI-Bambin et ALI-Explorateur, les adultes reçoivent quatre livres pour enfants à chaque formation. Les livres donnés aux éducatrices qui s'adressent à un groupe d'enfants ( $n=4$ à 6 ) ont un plus grand format que ceux donnés aux parents. Les livres comportent peu de texte et sont adaptés à chaque volet des programmes. Les adultes ont le choix d'utiliser chacun des livres aussi souvent qu'ils le désirent ou d'autres, appropriés aux activités ALI. 


\section{Instruments de mesure}

\section{Développement cognitif de l'enfant}

Jusqu'à l'âge de 42 mois et 15 jours, chaque enfant est évalué à l'aide de l'échelle mentale du Bayley (Bayley, 1993) avant le début des programmes et 6 mois plus tard. L'échelle mentale du Bayley donne des résultats normalisés de développement (Index de Développement Mental : IDM) dont la moyenne est 100 et l'écart type 15. Elle possède de bonnes qualités psychométriques. Les coefficients de fidélité testretest sont 0,83 pour les enfants âgés de 1 à 12 mois et 0,91 pour ceux âgés de 24 à 36 mois. Une supervision étroite assure la fidélité de l'évaluation. Une assistante, étudiante au doctorat, examine les protocoles et vérifie la justesse des cotations. Au préalable, les évaluatrices reçoivent un entraînement rigoureux à l'administration et à la cotation de l'échelle du Bayley. En plus de l'IDM, nous calculons trois autres résultats. Nous obtenons ces résultats en regroupant, dans trois sous-échelles, tous les items de l'échelle mentale selon qu'ils évaluent plus spécifiquement le langage expressif, le langage réceptif ou les habiletés non-verbales. Les trois résultats se calculent selon le rapport d'items réussis cumulés par l'enfant sur le nombre total d'items cumulés de la sous-échelle en question. Cette approche s'inspire des méthodes de Dale, Bates, Reznick et Morisset (1989) et Reznick, Corley, Robinson et Matheny (1997) (Pour le détail et la validation de la répartition des items de l'échelle mentale dans les trois sous-échelles, voir Verreault, Malcuit, \& Pomerleau, 2005; Verreault, Pomerleau, Malcuit, \& Séguin, 2001).

À partir de 42 mois et 16 jours, l'Échelle d'intelligence Stanford-Binet (Thorndike, Hagen, \& Sattler, 1986) sert à évaluer les enfants. Comme le Bayley, elle est administrée avant le début du programme et 6 mois plus tard. Cette mesure évaluative offre un résultat normalisé de quotient intellectuel (QI) dont la moyenne est 100 et l'écart type 16. La corrélation test-retest est 0,91 à l'âge de 5 ans. La cotation de l'échelle pour les enfants de 2 à 6 ans permet d'établir un résultat normalisé en distribuant les huit sous-tests en deux facteurs: compréhension verbale et raisonnement non-verbal/visualisation. Les évaluatrices reçoivent un entraînement préalable à l'administration du test de façon à standardiser la passation. Une assistante, étudiante au doctorat, examine les protocoles et vérifie la justesse des cotations pour s'assurer d'un calibrage adéquat. De plus, pour 16,04\% des évaluations, deux personnes font indépendamment les cotations; le coefficient d'accord (Kappa) est 0,98.

\section{Journaux de lecture et appels téléphoniques}

Les adultes remplissent des journaux hebdomadaires pendant trois semaines à partir de chacune des formations jusqu'à la visite de consolidation. Ces journaux, sous forme de calendrier, colligent l'information sur la fréquence des activités ALI et leur durée. De plus, des appels téléphoniques mensuels permettent d'examiner avec l'adulte l'application des techniques et de recueillir l'information sur la fréquence et la durée des sessions. Une grille de suivi compile ces informations. Les journaux et les appels téléphoniques visent à s'assurer de la conformité des adultes aux programmes. Les données provenant des appels téléphoniques sont mises en relation 
avec les données des journaux de lecture. Les analyses montrent des corrélations significatives entre les données des appels et des journaux remplis par les parents (fréquences : $r=0,45, p<0,001$; durées : $r=0,54, p<0,001$ ). Les sessions de lecture sont plus fréquentes selon les appels téléphoniques que selon les journaux, $t(67)=$ $3,89, p<0,001$. Les analyses des durées ne montrent pas de différence entre les appels et les journaux. Pour les éducatrices, les fréquences et les durées ne varient pas entre les journaux et les appels. À la suite de ces résultats et parce que nous possédons un plus grand nombre de données provenant des appels téléphoniques, nous utilisons ces dernières pour décrire la conformité des adultes aux programmes ALI.

\section{Indices socio-démographiques et mode de garde}

À la première évaluation de l'enfant, un questionnaire collige des informations sur les caractéristiques socio-démographiques des familles et la fréquentation d'un milieu de garde par l'enfant. De ce questionnaire, nous tirons les informations suivantes : âge des parents, niveau de scolarité et revenu, statut civil, type de milieu de garde et durée de fréquentation.

\section{Résultats}

L'évaluation de l'impact d'ALI porte sur le développement cognitif des enfants dans les trois programmes aux deux moments de mesure, puis selon leur contexte d'implantation. Nous examinons aussi les mesures d'impact selon l'instrument utilisé. Des analyses supplémentaires sur les trois résultats à l'échelle mentale du Bayley et sur les deux facteurs du Stanford-Binet permettent de distinguer l'effet des programmes sur les habiletés cognitives langagières et non-langagières des enfants. Enfin, une dernière partie décrit la conformité des adultes aux demandes des programmes ALI, selon les rapports des parents et des éducatrices.

\section{Impact d'ALI selon les programmes}

La Figure 1 présente les moyennes des résultats normalisés de développement cognitif des enfants selon les trois programmes aux deux moments d'évaluation. Une analyse de la variance à mesures répétées avec les facteurs programmes (3) et moments de mesure (2) montre que, dans l'ensemble, les enfants font des gains d'un moment de mesure à l'autre, $F(1,109)=11,71, p<0,01$, (pré : $M=98,97, E ́ T=9,79$; post : $M=101,22, E ́ T=9,10)$. L'interaction programmes par moment, $F(2,109)=4,33$, $p<0,05$, indique que l'amélioration varie selon les programmes. L'analyse des effets simples révèle que la hausse des résultats entre les deux moments de mesure s'explique surtout par les gains des enfants des programmes ALI-Bébé, $\mathrm{t}(36)=-2,67$, $\mathrm{p}<0,05$, et ALI-Bambin, $t(30)=-3,15, p<0,01$. Les enfants du programme ALIExplorateur ne font pas de gain entre les deux moments de mesure, $t(43)=0,39$, $p=0,70$. 
Figure 1 : Résultats de développement selon les programmes aux deux moments de mesure (les lignes verticales représentent les erreurs types des moyennes).

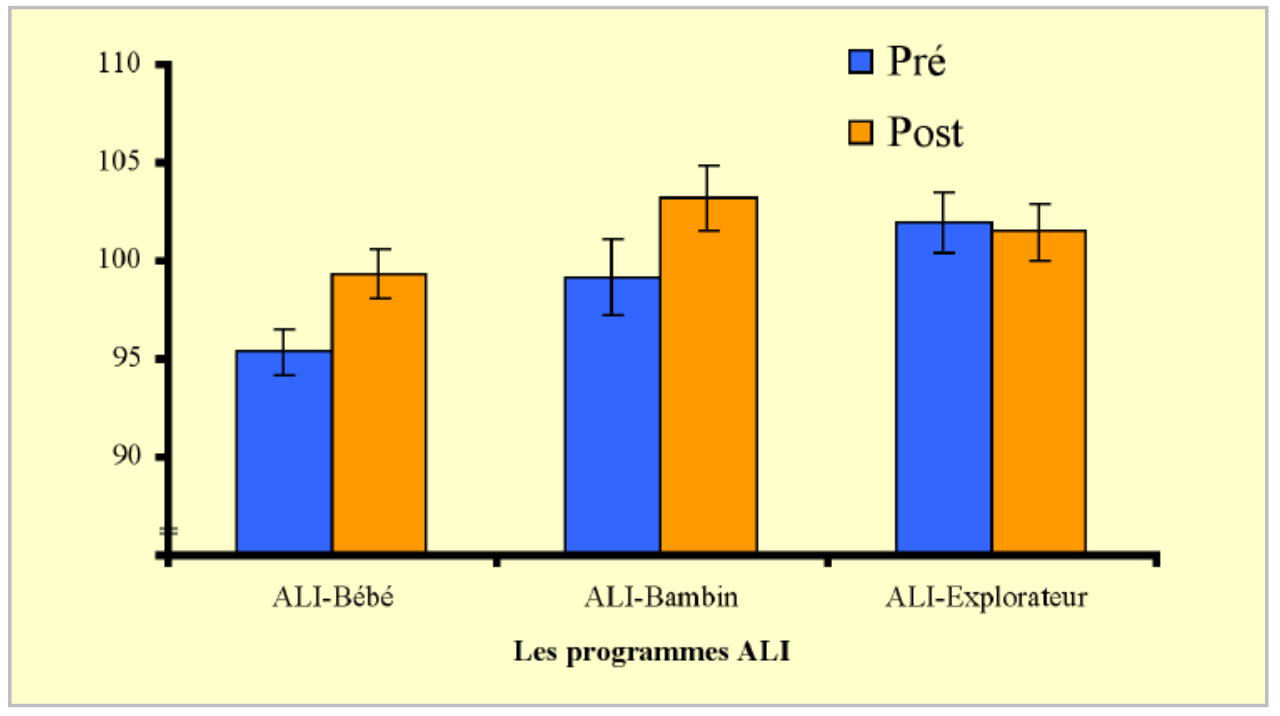

\section{Impact d'ALI selon les contextes d'implantation}

Les programmes ALI se réalisent à domicile, en milieu de garde ou dans les deux contextes. L'analyse de la variance à mesures répétées avec les facteurs contextes d'implantation (3) et moments de mesure (2) indique encore l'effet principal du temps, $F(1,109)=7,69, p<0,01$. Elle ne montre pas de différence entre les contextes d'implantation, $F(2,109)=0,001, p=0,99$, ni d'effet d'interaction, $F(2,109)=0,41$, $p=0,66$. Les résultats des enfants augmentent d'un moment de mesure à l'autre dans tous les contextes d'implantation, y compris celui où les programmes sont réalisés à la fois à domicile et en milieu de garde.

\section{Impact d'ALI selon les tests}

L'évaluation de l'impact des programmes ALI sur le développement cognitif des enfants se fait avec deux tests normalisés. Nous avons examiné si la mesure d'impact varie selon l'instrument utilisé. L'analyse à mesures répétées avec les facteurs tests (2) et moments de mesure (2) fait ressortir l'effet temps, $F(1,110)=5,46, p<0,05$, et une interaction tests par moment, $F(1,110)=6,70, p<0,05$. Les gains ne sont pas les mêmes selon le test utilisé (Bayley: 3,72 points; Stanford-Binet: -0,19 points). L'analyse des effets simples révèle une différence significative entre les deux moments de mesure pour les résultats à l'échelle mentale du Bayley, $t(69)=-3,84$, $p<0,001$, mais pas pour les résultats à l'Échelle d'intelligence Stanford-Binet, $t(41)=$ $0,17, p=0,86$. L'absence de différence entre les résultats du Stanford-Binet s'explique par le facteur programme. Sur les 42 évaluations réalisées à l'aide du Stanford-Binet, 40 portent sur des enfants ALI-Explorateur. D'après les analyses précédentes, les enfants touchés par ce programme ne font pas de gains. 


\section{Impact d'ALI sur les habiletés cognitives langagières et non-langagières}

Les programmes ALI visent de façon particulière le développement langagier des jeunes enfants. Pour vérifier cet effet, des analyses portent sur les trois résultats dérivés de l'échelle mentale du Bayley. Nous examinons de façon distincte les résultats des enfants ALI-Bébé et ALI-Bambin. Ces analyses distinctes s'expliquent d'abord par l'intérêt de comparer l'impact de chaque programme sur chacune des sous-échelles. Également, le répertoire verbal des nourrissons est très différent de celui des enfants plus âgés. L'acquisition du langage constitue l'habileté principale développée au cours de la deuxième année de vie des enfants (Edwards, 1995). Ainsi, les items langagiers occupent une plus grande place dans l'évaluation cognitive des enfants ALI-Bambin. Comme ils sont peu nombreux, les quatre enfants ALIExplorateur évalués avec l'échelle mentale du Bayley sont regroupés avec les enfants ALI-Bambin.

Pour ALI-Bébé, l'analyse de la variance à mesures répétées montre un effet principal sous-échelles, $F(2,72)=6,25, p<0,01$, et un effet d'interaction sous-échelles par moments, $F(2,72)=26,75, p<0,001$ (voir Figure 2). L'analyse des effets simples indique une différence significative entre les moments de mesure à deux des souséchelles. À la sous-échelle langage réceptif, les résultats diminuent entre les deux moments, $t(36)=4,79, p<0,001$, tandis qu'à la sous-échelle habiletés non-verbales les résultats augmentent, $t(36)=-7,05, p<0,001$. Les résultats de langage expressif ne diffèrent pas d'un moment de mesure à l'autre, $t(36)=0,02, p=0,99$. Les corrélations entre les résultats aux deux moments sont faibles et non significatives pour les deux sous-échelles verbales du Bayley, $r=0,15, p=0,38$, pour la sous-échelle expressive, et, $r=0,19, p=0,27$, pour la réceptive. Par contre, une corrélation positive entre les résultats aux deux moments à la sous-échelle d'habiletés non-verbales, $r=0,41, p<0,05$, souligne la relative stabilité dans le temps des scores cognitifs non-verbaux des enfants ALI-Bébé.

Pour ALI-Bambin, l'analyse de la variance à mesures répétées des résultats aux trois sous-échelles montre des effets principaux des sous-échelles, $F(2,64)=86,90$, $p<0,001$, du moment, $F(1,32)=19,09, p<0,001$, et un effet d'interaction souséchelles par moment, $F(2,64)=3,60, p<0,05$ (voir Figure 2). Les analyses d'effets simples indiquent une hausse significative des scores du langage expressif, $t(32)=$ $-4,47, p<0,001$, et des habiletés non-verbales des enfants, $t(32)=-3,12, p<0,01$, ainsi qu'une hausse marginale à la sous-échelle du langage réceptif, $t(32)=-1,74, p=0,09$. Les corrélations entre les résultats aux deux moments de mesure sont significatives pour les trois sous-échelles : $r=0,45, p<0,01$, pour l'expressive, $r=0,41, p<0,05$, pour la réceptive et, $r=0,39, p<0,05$, pour la non-verbale. 
Figure 2 : Proportions d'items réussis aux trois sous-échelles du Bayley aux deux moments de mesure.

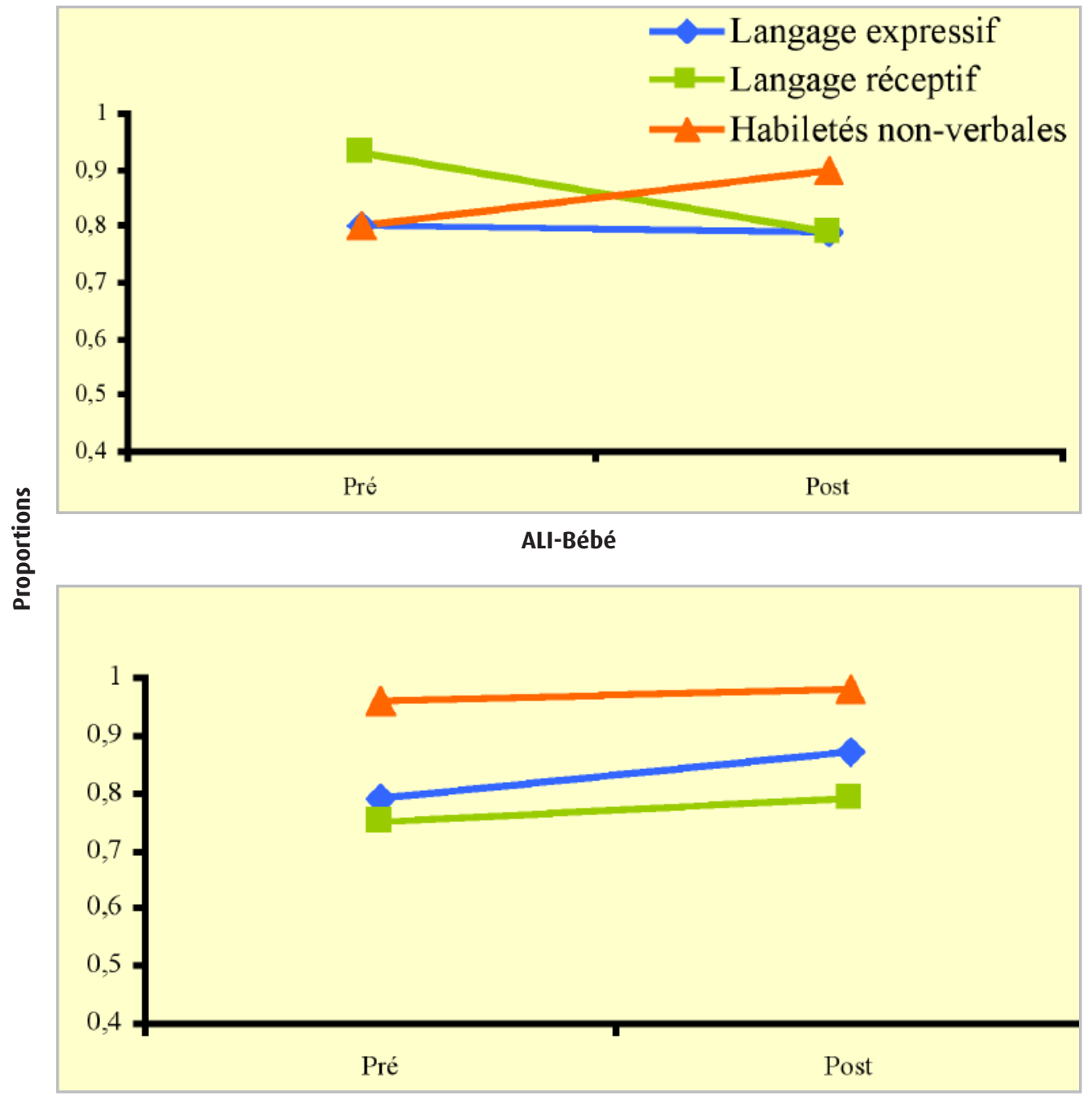

ALI-Bambin

Chez les enfants ALI-Explorateur (et pour les 2 enfants ALI-Bambin évalués avec le Stanford-Binet), les analyses supplémentaires pour distinguer l'effet sur les habiletés cognitives langagières portent sur les résultats normalisés aux deux facteurs du Stanford-Binet. Dans l'ensemble, les résultats aux facteurs compréhension verbale et raisonnement non-verbal/visualisation ne changent pas d'un moment de mesure à l'autre, $F(1,41)=1,01, p=0,32$. L'interaction moments par facteurs n'est pas significative, $F(1,41)=0,11, p=0,74$. Les enfants ont globalement des résultats supérieurs au facteur non-verbal/visualisation qu'au facteur verbal, $F(1,41)=20,62, p<0,001$ (compréhension verbale: $M=96,61$; raisonnement non-verbal/visualisation : $M=$ 102,68). 


\section{Conformité des adultes aux programmes}

Les données de conformité provenant des appels téléphoniques confirment que les parents et les éducatrices ont respecté les demandes des programmes (au moins 3 périodes hebdomadaires de 10 à 15 minutes chacune). Les activités d'ALI-Bébé ne sont pas quantifiées, car elles s'intègrent à la routine quotidienne de la famille ou du CPE. Pour le programme ALI-Bambin, les parents rapportent faire plus de 4 séances de lecture hebdomadaires d'une durée moyenne de plus de 12 minutes. Les parents du groupe ALI-Explorateur rapportent aussi faire de la lecture de façon régulière (plus de 3 périodes par semaine), pendant plus de 16 minutes chaque fois. Les analyses montrent que les parents d'ALI-Explorateur rapportent des durées plus longues que les parents d'ALI-Bambin, $\mathrm{F}(1,31)=5,63, \mathrm{p}<0,05$.

En milieu de garde, les éducatrices des programmes ALI-Bambin et ALIExplorateur réalisent aussi plus de 3 séances de lecture par semaine pour une durée moyenne au-delà de 14 minutes. Les analyses de la variance ne montrent pas de différence de fréquence ni de durée entre les éducatrices ALI-Bambin et les éducatrices ALI-Explorateur.

Des analyses supplémentaires comparent les fréquences d'activités ALI et les durées moyennes rapportées par les parents et par les éducatrices. Les fréquences et durées des activités ne diffèrent pas selon les parents ou les éducatrices et ce, pour les deux programmes.

\section{Discussion}

Nos résultats montrent que les enfants font des gains significatifs aux tests normalisés après six mois d'intervention. Cependant, les programmes n'ont pas tous la même efficacité. Ce sont surtout ALI-Bébé et ALI-Bambin qui entraînent des hausses de résultats normalisés. À notre surprise, la grandeur des gains ne diffère pas d'un contexte d'implantation à l'autre, ni lorsque les programmes sont réalisés à la fois à domicile et en milieu de garde. Finalement, nos résultats révèlent que l'intervention entraîne essentiellement des gains aux mesures de langage expressif et d'habiletés non-verbales. La discussion s'organise autour de ces constatations. Nous discutons également de la conformité des adultes et terminons en présentant les limites de la recherche.

Tout d'abord, même si globalement ALI entraîne des gains de points chez les enfants, les trois programmes n'ont pas tous un impact significatif. L'augmentation des résultats normalisés se retrouve essentiellement chez les enfants qui ont suivi les programmes ALI-Bébé et ALI-Bambin. Les enfants du programme ALI-Explorateur ne haussent pas leurs résultats d'un moment de mesure à l'autre. Nous pouvons avancer quatre hypothèses pour expliquer cette absence de changement : une intervention de type ALI ne serait efficace qu'avec des enfants plus jeunes, l'instrument d'évaluation ne parviendrait pas à détecter le changement, l'implantation du programme ALI-Explorateur aurait manqué d'intensité et, finalement, son contenu serait insuffisant pour hausser le développement cognitif d'enfants âgés de 3 à 5 ans. 
Nos résultats vont dans le sens des études qui montrent que les effets de la lecture sont plus importants lorsque les enfants sont plus jeunes (Bus et al., 1995; DeBaryshe, 1993; Scarborough \& Dobrich, 1994). Les chercheurs affirment que des programmes introduits à un plus jeune âge produisent de meilleurs effets (Ramey \& Ramey, 1998). Cependant, ils ne mentionnent pas qu'il existe une période sensible après laquelle des interventions peuvent être trop tardives. Si le plus tôt s'avère préférable, il n'est jamais trop tard pour intervenir. Il est possible d'obtenir des résultats positifs avec différents programmes chez des enfants âgés de 3 ans et plus (Barnett, 1995). L'âge ne nous paraît donc pas le principal facteur explicatif de l'absence d'efficacité du programme ALI-Explorateur.

Il est possible que le Stanford-Binet, utilisé comme instrument d'évaluation des enfants à partir de 3 ans et demi, soit moins apte à détecter les effets du programme ALI-Explorateur. Le Stanford-Binet propose un ensemble diversifié de tâches qui permettent de mesurer le niveau général du développement cognitif de l'enfant (Thorndike et al., 1986). Pour sa part, le programme ALI-Explorateur favorise l'acquisition de notions, telles le temps, les grandeurs, les similitudes, les couleurs, la numération, les animaux, les parties du corps, les formes, etc. Ces connaissances conceptuelles sont spécifiques et forment une partie du fonctionnement cognitif global de l'enfant. Il est possible que le Stanford-Binet soit trop général pour détecter des changements au niveau des connaissances conceptuelles visées par ALIExplorateur.

Pour les participants ALI-Explorateur, nous avons implanté un volet préparatoire (volet Début) pour initier les adultes aux techniques de lecture interactive proprement dites. Ce volet correspond au dernier d'ALI-Bambin. En examinant de près les données d'implantation, nous constatons que 24 des 44 enfants ALI-Explorateur ont surtout eu le volet Début entre leurs deux moments de mesure; ils n'ont pas bénéficié du contenu des volets spécifiques du programme ALI-Explorateur durant un minimum de trois mois. La courte durée d'implantation du programme et la prédominance du dernier volet d'ALI-Bambin auprès d'enfants plus âgés peuvent expliquer, en partie, l'absence de gains chez ces enfants.

Le programme ALI-Explorateur permet à l'enfant d'apprendre et d'utiliser un vocabulaire de plus en plus élaboré pour nommer, décrire et situer, dans l'espace et le temps, les personnes, les objets et les événements qui l'entourent. Cependant, il ne présente pas d'activités pour favoriser l'acquisition des nombreuses habiletés cognitives en émergence chez des enfants de 3 à 5 ans, telles la mémoire, le raisonnement logique, etc. Le Stanford-Binet mesure ces habiletés. Dans une nouvelle version (Malcuit et al., 2003), les concepteurs ont enrichi le contenu du programme ALIExplorateur pour mieux intégrer ces différentes habiletés. La nouvelle version devrait pouvoir entraîner des effets décelables avec le Stanford-Binet.

Les résultats des enfants augmentent après six mois de programme, mais la grandeur des gains ne diffère pas selon qu'il soit mené à domicile ou en milieu de garde, ni même, plus étonnant, qu'il soit réalisé aux deux endroits. Nos données de conformité peuvent expliquer, en partie, le fait que les programmes à la maison aient autant d'impact sur les habiletés cognitives que ceux réalisés en milieu de garde. Les parents 
et les éducatrices ont consacré un temps égal à faire les activités. Ainsi, les enfants sont autant exposés aux contenus ALI à domicile qu'en milieu de garde. Les qualités propres aux deux contextes expliquent aussi l'efficacité équivalente de l'intervention.

L'implantation des activités ALI à domicile offre ses avantages: activités individuelles, généralisation à d'autres contextes et grande variabilité des stimulations par la famille. Dans un contexte dyadique, le parent est en mesure d'adapter les techniques de lecture interactive à l'intérêt et aux capacités de l'enfant. L'ajustement des façons de faire du parent au niveau de l'enfant permet de développer efficacement ses habiletés cognitives (Lonigan \& Whitehurst, 1998). Aussi, les familles rapportent avoir généralisé leur application des techniques ALI au cours de multiples activités quotidiennes (lors des repas, des soins, des promenades, etc.). Les interventions ont davantage d'impact lorsque leur contenu touche un large éventail d'activités (Hart \& Risley, 1995). Également, nous formions aux programmes les diverses personnes en interaction avec l'enfant. Les grands-parents ou la fratrie pouvaient réaliser des activités ALI avec l'enfant de façon quotidienne, ce qui lui permettait de profiter d'une plus grande variété de stimulations. La réalisation d'activités ALI peut être bien intégrée dans le quotidien des familles. Ceci explique les gains des enfants qui ont le programme à domicile.

Le milieu de garde permet aussi, et peut-être davantage, une régularité des stimulations grâce à l'intégration des activités des programmes à la routine quotidienne. La lecture traditionnelle est souvent incluse dans la programmation des milieux de garde. Elle fait partie des réglementations gouvernementales (Ministère de la famille et de l'enfance, 1997). Il devient facile d'y appliquer les techniques ALI. De plus, en milieu de garde, les activités ALI se réalisent en petit groupe. La situation de groupe crée de plus grandes exigences pour l'enfant et constitue un contexte d'émulation favorable à son développement. Comparativement à ce qui se passe à la maison, où le parent comprend les approximations langagières de l'enfant et ajuste ses propres verbalisations au niveau de ce dernier, le groupe, avec des personnes moins familières, est plus exigeant. Dans ce contexte, l'enfant doit préciser davantage ses expressions verbales pour se faire comprendre. Le contexte social du milieu de garde favorise l'émulation et les conduites d'échange verbal lors des nombreux contacts de l'enfant avec les adultes et ses pairs (National Institute of Child Health and Human Development Early Child Care Research Network, 2000). Aussi, par leur formation, les éducatrices comprennent l'importance de la lecture interactive et du rôle qu'elles jouent pour favoriser le développement de l'enfant. La réalisation des activités ALI peut être intense en milieu de garde (Perna, 2003), ce qui explique les gains obtenus.

Finalement, nous supposions des gains plus importants chez les enfants qui reçoivent les programmes à la fois à domicile et en milieu de garde, car ils sont plus fréquemment exposés aux activités ALI. De façon constante, la recherche révèle une relation positive entre l'intensité avec laquelle on applique des programmes et l'importance de leurs effets (Barnett, 1995; Ramey \& Ramey, 1998). Il est possible que l'absence de différence entre les groupes s'explique par le très petit nombre d'enfants à avoir combiné les deux modalités d'application. En effet, seulement 13 enfants, dont 7 dans ALI-Explorateur, constituent le groupe domicile+milieu de garde. Consi- 


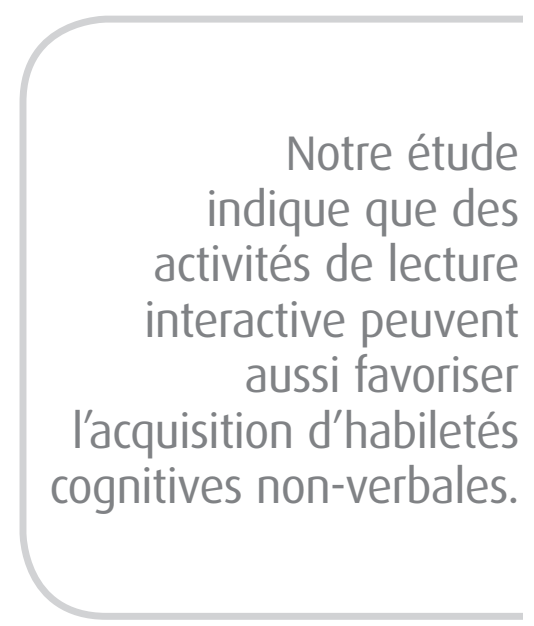

dérant la relative inefficacité du programme ALI-Explorateur, il reste peu d'enfants dans ce groupe pour dégager des gains différentiels. Des recherches futures devront établir des comparaisons entre l'intensité des programmes ALI et leurs effets.

Les programmes ALI ont un impact différent sur les habiletés cognitives langagières et non-langagières des enfants. Évidemment, les activités de stimulation ALIBébé produisent des gains au niveau des habiletés non-verbales, fonctions dominantes pour des enfants âgés entre 0 et 15 mois. Chez des enfants un peu plus âgés, les études sur la lecture dialogique rapportent essentiellement des gains en langage expressif (Lonigan \& Whitehurst, 1998; Valdez-Menchaca \& Whitehurst, 1992; Whitehurst et al., 1988; Whitehurst, Arnold et al., 1994; Whitehurst, Epstein et al., 1994). Les gains en langage réceptif sont plus mitigés. Nos résultats vont dans le même sens pour les enfants ALI-Bambin. Les analyses indiquent une hausse significative des résultats au langage expressif, ainsi qu'une hausse marginale au langage réceptif. Or, aucune des études antérieures ne présente de mesures d'habiletés nonverbales. Notre étude indique que des activités de lecture interactive peuvent aussi favoriser l'acquisition d'habiletés cognitives non-verbales entre 15 et 36 mois. Ce résultat peut s'expliquer par le cadre dans lequel les activités ALI-Bambin se déroulent. Pendant la lecture d'imagiers, l'adulte attire l'attention de l'enfant sur les images, ce qui l'aide à développer des habiletés non-verbales, telles la discrimination visuelle, l'attention et la coordination visuo-spatiale. Enfin, chez les enfants ALIExplorateur, les analyses sur les facteurs compréhension verbale et raisonnement non-verbal/visualisation du Stanford-Binet montrent que les résultats normalisés ne changent pas. Même en distinguant les habiletés cognitives langagières et nonlangagières, le programme ALI-Explorateur semble inefficace.

Les données de conformité révèlent que les parents ont respecté les demandes des programmes. En milieu de garde, nous savons que les éducatrices font les activités ALI régulièrement, mais nous ne savons pas si les enfants que nous évaluons sont toujours présents. Nous n'avons pas comptabilisé les présences des enfants, ni leur participation individuelle lors des sessions ALI. Les recherches futures devraient s'assurer d'avoir des mesures, toutefois intrusives, qui répertorient les enfants participant à chacune des activités de lecture interactive. Il serait alors possible de mettre en lien les fréquences des activités et les gains chez les enfants.

\section{Limites et contributions de la recherche}

Il existe des limites à la présente recherche. Tout d'abord, elle ne repose pas sur un devis expérimental avec assignation au hasard aux programmes et à une condition de contrôle. Les résultats se basent sur des tests standardisés avec des résultats normalisés. Il faut situer le contexte de l'étude pour expliquer ce choix méthodologique. Les programmes ALI s'insèrent dans le cadre d'un large projet, l'initiative 1,2,3GO!, implanté dans une municipalité rurale de la Montérégie. Dans ce contexte, nous avons rencontré des contraintes conceptuelles et financières. La philosophie de l'initiative 1,2,3GO! réclame un accès universel à tout ce que la communauté offre, dont les programmes ALI. Ceci empêche donc une vraie expérimentation avec assignation au hasard d'enfants aux programmes et d'autres à une condition de contrôle. 
Dans ce cas, nous aurions pu recourir à un schème quasi-expérimental, en trouvant une communauté de comparaison aussi équivalente que possible à la communauté expérimentale pour y recruter les enfants de la condition de contrôle. Cependant, l'initiative 1,2,3GO! a choisi le territoire où nous avons implanté les programmes ALI en fonction de facteurs de risques socio-démographiques particuliers: sousscolarisation, isolement des familles, éloignement des services, absence de transport en commun, etc. (Pomerleau et al., 1997). Il est toujours possible (mais très difficile) de trouver un territoire de comparaison présentant des caractéristiques similaires (Denis, Malcuit, \& Pomerleau, accepté). Il faut souligner que l'implantation et l'évaluation des programmes ALI se sont déroulées sur une période de quatre ans. On constate généralement que même si au départ les territoires expérimental et de contrôle paraissent à peu près équivalents, les communautés évoluent durant une si longue période de temps, et habituellement de façon différente. Le bon choix du début ne garantit donc pas la ressemblance à plus long terme.

Dans le cadre de ces contraintes, nous avons opté pour des tests normalisés reconnus. Ils situent la performance de chaque enfant par rapport à celles des enfants de son groupe d'âge. En l'absence d'événement particulier systématique entre deux moments de mesure, un enfant devrait obtenir des résultats normalisés similaires à des évaluations successives. S'il y a hausse significative des résultats d'une évaluation à l'autre, on peut l'attribuer à un événement particulier : les programmes ALI ou une variable historique, telle la mise en place d'un autre programme dans la communauté qui toucherait tous les enfants. Outre ALI, nous n'avons identifié aucun événement systématique susceptible d'expliquer des hausses de résultats normalisés chez les enfants de deux des trois programmes. S'il y avait eu une variable inconnue dans l'histoire de la communauté, on aurait pu s'attendre à ce qu'elle entraîne aussi des hausses de résultats chez les enfants ALI-Explorateur. Si la grandeur des gains significatifs des enfants ALI-Bébé et ALI-Bambin peut paraître modérée malgré tout, il faut souligner que les programmes inversent la tendance à la diminution progressive des scores de développement qui paraît de façon générale dans les milieux socio-économiques moins favorisés (Lacroix et al., 2002).

Également, nous avons poussé un peu plus loin les analyses statistiques pour confirmer l'impact d'ALI. Pour être inclus dans l'étude, les enfants devaient participer aux programmes un minimum de trois mois. Lorsque les dossiers des formatrices ne permettaient pas d'établir la participation active et intensive des adultes, les données des enfants $(n=38)$ n'étaient pas incluses dans les analyses d'impact. Des analyses sur les données de ces enfants montrent que leurs résultats de développement n'augmentent pas d'un moment de mesure à l'autre, $t(37)=-1,15, p=0,26$. Quand les enfants reçoivent peu les programmes, leurs v normalisés ne changent pas de façon significative (Verreault, 2005).

Nous avons aussi examiné les données des participants en formant deux cohortes de nombre égal d'enfants selon la période où ils ont commencé pour chacun des trois programmes. Des analyses de la variance à mesures répétées avec les facteurs cohortes (2) et moments de mesure (2) portent sur les données des enfants de chaque programme. Ces analyses confirment nos conclusions antérieures. Elles 
montrent un effet moments de mesure et aucun effet d'interaction cohortes par moments de mesure pour les programmes ALI-Bébé et ALI-Bambin. Elles révèlent aussi l'absence d'effet du programme ALI-Explorateur dans la modification des résultats de développement des enfants (Verreault, 2005).

L'évaluation des programmes ALI ne comporte pas de mesure différée. Il serait intéressant de vérifier si les effets obtenus à court terme se maintiennent jusqu'à la maternelle et au-delà, si les effets sont plus importants à la suite d'un programme de plus longue durée ou, encore, si des effets qui ne sont pas immédiats apparaissent quelques temps après. Les effets bénéfiques de la lecture dialogique persistent jusqu'à la fin de la maternelle (Whitehurst et al., 1999). Avec une action mobilisant la communauté, on peut espérer que les programmes ALI contribueront au développement cognitif et langagier ultérieur des enfants de cette municipalité.

Notre étude souligne l'importance des familles et des milieux de garde pour la stimulation cognitive en contexte de lecture. Plus particulièrement, les résultats montrent que des Activités de Lecture Interactive participent au développement cognitif et langagier des enfants durant les années préscolaires. Les programmes ALI respectent les objectifs et principes de base du programme éducatif des centres de la petite enfance du Ministère de la famille et de l'enfance (1997). Ils font d'ailleurs l'objet d'une implantation dans l'ensemble des milieux de garde de la Montérégie.

\section{Références bibliographiques}

ALEXANDER, K. L., \& ENTWISLE, D. R. (1988). Achievement in the first 2 years of school: Patterns and processes. Monographs of the Society for Research in Child Development, 53(2, Serial No. 218).

BARNETT, S. W. (1995). Long-term effects of early childhood programs on cognitive and school outcomes. The Future of Children, 5, 25-50.

BAYLEY, N. (1993). Bayley Scales of Infant Development, Second Edition. San Antonio, TX: The Psychological Corporation. Bus, A. G., van IJzendoorn, M. H., \& Pellegrini, A. D. (1995). Joint book reading makes for success in learning to read: A meta-analysis on intergenerational transmission of literacy. Review of Educational Research, 65, 1-21.

Carnegie Foundation for the Advancement of Teaching. (1991). Ready to learn: A mandate for the nation. Princeton, $\mathrm{NJ}$ : Auteur.

CRAIN-THORESON, C., \& DALE, P. S. (1992). Do early talkers become early readers? Linguistic precocity, preschool language, and emergent literacy. Developmental Psychology, 28, 421-429. 
DALE, P. S., BATES, E., REZNICK, J. S., \& MORISSET, C. (1989). The validity of a parent report instrument of child language at twenty months. Journal of Child Language, 16, 239-249.

DEBARYSHE, B. D. (1993). Joint picture-book reading correlates of early oral language skill. Journal of Child Language, 20, 455-461.

DENIS, E., MALCUIT, G., \& POMERLEAU, A. (accepté). Évaluation des impacts de l'initiative communautaire 1,2,3GO! sur le développement et le bien-être des tout-petits et de leur famille. Éducation et Francophonie.

EDWARDS, C. P. (1995). Parenting toddlers. Dans M. H. Bornstein (Éd.), Handbook of parenting, Vol. 1: Children and parenting Hillsdale, NJ : Lawrence Erlbaum Associates, 41-63.

HART, B. (1991). Input frequency and children's first words. First Language, 11, 289-300.

HART, B., \& RISLEY, T. R. (1995). Meaningful differences in the everyday experience of young american children. Baltimore, MD : Paul H. Brookes.

HOFF-GINSBERG, E. (1985). Some contributions of mothers' speech to their children's syntactic growth. Journal of Child Language, 12, 367-385.

HOFF-GINSBERG, E. (1991). Mother-child conversation in different social classes and communicative settings. Child Development, 62, 782-796.

HUTTENLOCHER, J., HAIGHT, W., BRYK, A., SELTZER, M., \& LYONS, T. (1991). Early vocabulary growth: Relation to language input and gender. Developmental Psychology, 27, 236-248.

LACROIX, V., POMERLEAU, A., \& MALCUIT, G. (2002). Properties of adult and adolescent mothers'speech, children's verbal performance and cognitive development in different socioeconomic groups: a longitudinal study. First Language, 22, 173-196.

LEWIS, C., \& GREGORY, S. (1987). Parents' talk to their infants: The importance of context. First Language, 7, 201-216.

LONIGAN, C. J., \& WHITEHURST, G. J. (1998). Relative efficacy of parent and teacher involvement in a shared-reading intervention for preschool children from low-income backgrounds. Early Childhood Research Quarterly, 13, 263-290.

LYYTINEN, P., LAAKSO, M.-L., \& POIKKEUS, A.-M. (1998). Parental contribution to child's early language and interest in books. European Journal of Psychology of Education, 13, 297-308.

MALCUIT, G., POMERLEAU, A., \& SÉGUIN, R. (2003). Activités de lecture interactive. Saint-Hubert: Les Éditions du Regroupement des centres de la petite enfance de la Montérégie. 
MCDONNELL, S. A., ROLLINS, P. R., \& FRIEL-PATTI, S. (2001, avril). Patterns of change in maternal/child discourse behaviors across repeated storybook readings. Communication présentée au congrès bisannuel de la Society for Research in Child Development (SRCD), Minneapolis, MN.

Ministère de la famille et de l'enfance. (1997). Programme éducatif des centres de la petite enfance. Québec: Les publications du Québec.

National Center for Early Development and Learning. (1998). Kindergarten transitions. NCEDL Spotlights, Number 1.

National Institute of Child Health and Human Development Early Child Care Research Network. (2000). The relation of child care to cognitive and language development. Child Development, 71, 960-980.

NINIO, A. (1992). The relation of children's single word utterances to single word utterances in the input. Journal of Child Language, 19, 87-110.

PERNA, I. (2003). Programme de lecture dialogique à la maison et en milieu de garde: évolution des conduites interactives de parents de deux milieux socio-économiques. Thèse de doctorat non-publiée. Université du Québec à Montréal, Montréal, Canada.

POMERLEAU, A., MALCUIT, G., LABERGE, D., \& FLYNN, L. (1997). Projet TLC 3 . Programme ALI. Demande de subvention, The Hincks Centre for Children's Mental Health \& The Lawson Foundation, Montréal.

RAMEY, C. T., \& RAMEY, S. L. (1998). Early intervention and early experience. American Psychologist, 53, 109-120.

REZNICK, J. S., CORLEY, R., ROBINSON, J., \& MATHENY, A. P. (1997). A longitudinal twin study of intelligence in the second year. Monographs of the Society for Research in Child Development, 62(1, Serial No. 249).

SCARBOROUGH, H. S., \& DOBRICH, W. (1994). On the efficacy of reading to preschoolers. Developmental Review, 14, 245-302.

SNOW, C. E. (1984). Parent-child interaction and the development of communicative ability. Dans R. L. Schiefelbusch, \& J. Pickar (Éd.), The acquisition of communicative competence (pp. 69-107). Baltimore, MD : University Park Press.

STEVENSON, H. W., \& NEWMAN, R. S. (1986). Long-term prediction of achievement and attitudes in mathematics and reading. Child Development, 57, 646-659.

STEVENSON, J., \& FREDMAN, G. (1990). The social environmental correlates of reading ability. Journal of Child Psychology and Psychiatry, 31, 681-698.

THORNDIKE, R. L., HAGEN, E. P., \& SATTLER, J. M. (1986). Échelle d'intelligence Stanford-Binet, Quatrième Édition. Montréal: Institut de recherches psychologiques. 
VALDEZ-MENCHACA, M. C., \& WHITEHURST, G. J. (1992). Accelerating language development through picture book reading: A systematic extension to mexican day care. Developmental Psychology, 28, 1106-1114.

VERREAULT, M. (2005). Programmes d'activités de lecture interactive et développement cognitif de jeunes enfants : mesures d'impact et comparaison d'échelles d'évaluation (Bayley et Stanford-Binet). Thèse de doctorat non-publiée. Université du Québec à Montréal, Montréal, Canada.

VERREAULT, M., MALCUIT, G., \& POMERLEAU, A. (2005). Évaluation du développement cognitif d'enfants d'âge préscolaire au moyen de deux échelles : échelle mentale du Bayley et Échelle d'intelligence Stanford-Binet. Manuscrit soumis pour publication.

VERREAULT, M., POMERLEAU, A., MALCUIT, G., \& SEGUIN, R. (2001). Regroupement des items de l'échelle mentale du Bayley (1993) en trois sous-échelles : langage expressif, langage réceptif, habiletés non-verbales. Document non-publié, Laboratoire d'étude du nourrisson, Université du Québec à Montréal, Canada.

WELLS, G. (1987). The learning of literacy. Dans B. Fillion, C. N. Hedley, \& E. C. DiMartino (Éd.), Home and school: Early language and reading Norwood, NJ : Ablex, 27-45.

WHITEHURST, G. J., ARNOLD, D. S., EPSTEIN, J. N., ANGELL, A. L., SMITH, M., \& FISCHEL, J. E. (1994). A picture book reading intervention in day care and home for children from low-income families. Developmental Psychology, 30, 679-689.

WHITEHURST, G. J., EPSTEIN, J. N., ANGELL, A. L., PAYNE, A. C., Crone, D. A., \& FISCHEL, J. E. (1994). Outcomes of an emergent literacy intervention in Head Start. Journal of Educational Psychology, 86, 542-555.

WHITEHURST, G. J., FALCO, F. L., LONIGAN, C. J., FISCHEL, J. E., DEBARYSHE, B. D., VALDEZ-MENCHACA, M. C., \& CAULFIELD, M. (1988). Accelerating language development through picture book reading. Developmental Psychology, 24, 552-559.

WHITEHURST, G. J., ZEVENBERGEN, A. A., CRONE, D. A., SCHULTZ, M. D., VELTING, O. N., \& FISCHEL, J. E. (1999). Outcomes of an emergent literacy intervention from Head Start through second grade. Journal of Educational Psychology, 91, 261-272.

\section{Notes des auteurs}

Cette recherche constitue une partie de la thèse de doctorat de la première auteure. Celle-ci a reçu une bourse du Conseil québécois de la recherche sociale (CQRS), du Fonds pour la formation de chercheurs et l'aide à la recherche du Québec (FCAR) et du Programme d'aide financière à la recherche et à la création (PAFARC). 
L'élaboration des programmes ALI et la recherche ont été rendues possibles grâce à des subventions du CQRS, du FCAR, du Conseil de recherches en sciences humaines du Canada (CRSH), de l'Institut de recherche pour le développement social des jeunes (IRDS) et de la fondation Lawson.

Nous tenons à remercier Renée Séguin, toutes les collaboratrices, les familles et leurs enfants pour leur contribution indispensable à l'étude.

Pour toute correspondance concernant cet article, s'adresser à Martine Verreault, Clinique des troubles de l'attention, Hôpital Rivière-des-Prairies, 7070 Boulevard Perras, Montréal, (Québec), Canada, H1E 1A4; courriel: martine.verreault.hrdp@ ssss.gouv.qc.ca 